\title{
An accurate setting for remapping process of foot plantar pressure
}

\begin{abstract}
This paper present a precise and detailed study for the touch area on the foot based on amplifier gain settings of individual sensors that are distributed over the plantar insole area, the idea is to fill the insole area with sensor size areas that satisfy covering almost the foot touch area. The procedure includes steps have to be implemented to achieve accurate reference values with respect to body weight. The values of individual sensor points have been evaluated for the both (Foot-Sensor-Insole-Ground) and (Foot-Sensor-Ground). It is found that, there is a wide range difference between the test points in each case and the existence of the insole reduced the pressure weight. A percentage value according to total body weight can be being assigned as the reference points in future studies.
\end{abstract}

Keyword: force sensor; Operation amplifier; Plantar pressure; Sensor processing 